DOI: $10.12731 / 2070-7568-2020-4-415-427$

УДК 004.89+336.71

\title{
ЦИФРОВАЯ ТРАНСФОРМАЦИЯ БИЗНЕС-ПРОЦЕССОВ КОММЕРЧЕСКИХ БАНКОВ ПОД ВЛИЯНИЕМ ТЕХНОЛОГИЙ ИСКУССТВЕННОГО ИНТЕЛЛЕКТА
}

\section{Терещенко С.Н.}

Технологии искусственного интеллекта сегодня являются сквозными технологиями ичифровой трансформации субъектов экономической деятельности, в том числе и банковского сектора. Коммерческие банки, за последнее десятилетие, прошли существенный путь трансформации от классических банков с филиальной сетью с менеджерско-кассирским обслуживанием до практически полного преврашения банка в онлайн-сервис с обработкой многих функций интеллектуальным агентом без участия человека. Банковский сектор стал одним из драйверов внедрения систем искусственного интеллекта. Для оченки ключевых этапов циифровой трансформации банковского сектора в мире можно использовать анализ крупнейших мировых соревнований по программированию и прикладному применению технологий искусственного интеллекта для задач банковской отрасли.

В статье рассматриваются технологические подходы по решению ключевых задач банковского дела в международных соревнованиях по программированию. Результаты соревнований анализируются как срез текущего запроса на технологии от банковского сектора и показатели уровня развития технологий искусственного интеллекта.

Цель-исследование хода проведения и результатов международных соревнований по применению технологий искусственного интеллекта в задачах банковского дела как метрики ичифровой трансформации бизнес-прочессов банковского сектора. 
Метод или методология проведения работы: в статье использовались приемы и инструменты системного анализа и обобщения.

Результаты: выявлены ключевые этапы циифровой трансформации бизнес-процессов банковского сектора за период 2010 2020 гг. и определен вектор развития технологий банковского дела.

Область применения результатов: полученные результаты целесообразно применять субъектами банковского сектора для выстраивания стратегии ичифровой трансформации бизнес-процессов.

Ключевые слова: искусственный интеллект; соревнования по программированию; Kaggle; компьютерное зрение; кредитный скоринг: бизнес-прочессы банковского сектора; ичировая трансформащчия.

\section{DIGITAL TRANSFORMATION \\ OF BUSINESS PROCESSES OF COMMERCIAL BANKS UNDER THE INFLUENCE OF ARTIFICIAL INTELLIGENCE TECHNOLOGIES}

\section{Tereshchenko S.N.}

Artificial intelligence technologies today are end-to-end technologies of digital transformation of economic entities, including the banking sector. Commercial banks over the past decade have gone through a transformation path up to the almost complete transformation of the bank into an online service of the human - intelligent agent bundle, where the role of a person has fewer and fewer functions. The banking sector has become one of the drivers of the introduction of artificial intelligence systems. To assess the key stages of the digital transformation of the banking sector in the world, you can use the analysis of the world's largest competitions in programming and the application of artificial intelligence technologies for the tasks of the banking industry. The article deals with technological approaches to solving key problems of banking in international programming competitions. The results of the competition are analyzed as a cross-section of the current request for 
technologies from the banking sector and indicators of the technological state of artificial intelligence technologies. Conducting an analysis on the tasks of international competitions, whose customers are not representatives of the banking sector, but which have a significant impact on banking business processes.

Purpose. Research of topics and results of international competitions on the use of artificial intelligence technologies in banking tasks as a metric of digital transformation of business processes in the banking sector.

Methodology in article method of system analysis, and also statistical methods of the analysis were used.

Results: the key stages of digital transformation of business processes of the banking sector for the period 2010-2020 years and the vector of development of banking technologies are received.

Practical implications the obtained results should be applied by the subjects of the banking sector to build a strategy for digital transformation of business processes.

Keywords: artificial intelligence; programming competitions; kaggle; computer vision; credit scoring: business processes of the banking sector; digital transformation.

\section{Введение}

Технологии искусственного интеллекта можно по праву считать одними из ключевых в общем тренде технологического развития человеческой цивилизации [5]. Они оказывают широкое влияние на многие экономико-социальные процессы, в том числе, и на банковскую сферу. Современные банки вынуждены проводить цифровую трансформацию своих бизнес-процессов [8] и структур, что делает их все более похожими не на типовой банк, а на высокотехнологичную ИТ-компанию [9]. Цифровая трансформация финансовой сферы, в том числе и банковской, рассмотрены в работах $[4,6$, 7]. Сущность цифровой трансформации банковской деятельности можно определить следующим. Цифровизация финансовой отрасли представляет собой сочетание двух тенденций: активного вторжения на финансовый рынок инновационных технологических компаний 
и освоения традиционными финансовыми организациями инновационных цифровых технологий [4, стр. 78].

Под технологиями искусственного интеллекта понимают функции человеческой деятельности, связанные с мозговой активностью, которые могут в какой-то мере заместить ИТ-технологии.

Принято разделять большой и малый искусственный интеллект. Под большим подразумевают полноценный искусственный разум, способный полностью заменить собой человека. Под малым понимают выполнение интеллектуальным агентом некоторых отдельных функций человеческого мозга, таких, как компьютерное зрение, распознавание речи, обработка текстовой информации.

Сегодня смело можно говорить о технологическом скачке в малом искусственном интеллекте. Совокупный набор этих отдельных функций уже стал причиной глобальной цифровой трансформации во всем мировом банковском секторе [9].

Первые серьезные разработки в области технологий искусственного интеллекта (нейронные сети) были проведены еще на рубеже 4050-х годов 20-го века. С тех пор технологический прогресс уверенно шел к увеличению доли отдельных замещаемых мозговых функций.

Банковский (и финансовый) сектор всегда был одним из основных потребителей и заказчиков технологий искусственного интеллекта. Сегодня высокотехнологичные банки являются одними из главных драйверов технологий искусственного интеллекта, наравне с ИТ-гигантами FAANG (англ. аббревиатура компаний Facebook, Apple, Amazon, Netflix и Google).

Выделяется отдельный термин - ФинТех - для определения использования информационных технологий в финансовой и банковской сфере [14].

\section{Цель исследования}

Коммерческие банки за последнее десятилетие прошли существенный путь трансформации от классических банков с филиальной сетью с менеджерско-кассирским обслуживанием до практически полного превращения банка в онлайн-сервис с обработкой 
многих функций интеллектуальным агентом без участия человека [1]. Для выделения ключевых вех оценки этапов и стадий цифровой трансформации можно выдвинуть следующую гипотезу: в качестве метрики динамики цифровой трансформации банковского сектора можно рассматривать задачи и результаты их решения в крупных международных соревнованиях по программированию в сфере искусственного интеллекта для предметной области банковского дела и смежных отраслей. Проверку гипотезы можно осуществить путем выявления связи между конкурсами по искусственному интеллекту в банковском деле и цифровой трансформацией банковского сектора.

\section{Материалы и методы}

В статье использовались приемы и инструменты системного анализа и обобщений. Проведена систематизация конкурсов по искусственному интеллекту в банковском деле. Анализ и корреляция задач и результатов проведения конкурсов, этапов осуществления цифровой трансформации банковского сектора.

\section{Результаты исследования и их обсуждения}

Сегодня Kaggle (https:/www.kaggle.com) - один из ключевых элементов в становлении, развитии и коммуникации специалистов по искусственному интеллекту. Это одна из мировых платформ для проведения соревнований по задачам сферы искусственного интеллекта. Платформа объединяет заказчиков, которыми зачастую выступают коммерческие организации, и специалистов разработчиков по всему миру. Заказчик подготавливает задачу, формирует набор данных, определяет метрику подсчета результата и, как правило, денежный приз за первые призовые места. По задачам данного конкурса можно коррелировать прогресс в сфере искусственного интеллекта. Разработчиками выступают тысячи программистов по всему миру. Таким образом, платформа Kaggle использует принципы краудсорсинга, что также является одним из признаков цифровой трансформации. Исследование опыта краудсорсинга в банковской сфере представлено в работе [3]. 
Рассмотрим цикл цифровой трансформации на примере конкурса Kaggle «Home Credit Default Risk».

Данный конкурс стал одним из знаковых соревнований банковского сектора. Постановщиком задания для программистов выступил банк Home Credit. В качестве задачи было предложено рассчитать вероятность возврата выданного заемщику кредита на основании анкетных данных собираемых банком.

Данные собраны банком на основе реальной информации о клиентах, но с проделанной операцией по полному обезличиванию. Машинным алгоритмам предстают обезличенные данные о клиенте с набором параметров: возраст, пол, доход, цель кредита и другими стандартными, которые заявитель вносит о себе при оформлении заявки на получение кредита.

Участникам предлагалось разработать алгоритмы, обучить их на открытых данных, которые свободно доступны на платформе Kaggle, и загрузить свое решение, которое еще пройдет проверку на закрытых данных. Итоговый результат публикуется на сайте Kaggle для общего соревновательного обзора. В качестве метрики результата выступил обобщенный показатель отклонения предсказанной алгоритмом вероятности возврата кредитов по заявлениям, от фактических данных, предоставленных банком.

Победители преодолели отметку 0,8 , что означает точность предсказания 80-ти процентов заявок на предмет возврата кредита.

Данный конкурс можно рассматривать как тренд, который шел в момент проведения конкурса во всем банковском секторе $[13,16]$. Расчет вероятности возврата кредита всегда была человеческой прерогативой. Работник, выполняющий функции по данной оценке, должен иметь высшее профильное образование и серьезный опыт работы. Нельзя поручить данную работу человеку без профессиональных навыков и подготовки.

Результат 80\% дает выбор для руководства, оставлять людей принимать решения о выдаче кредита, или доверить машинному алгоритму (и/или нейросетевым технологиях). Далее каждая финансовая организация принимает решение о степени и сроках цифро- 
вой трансформации. Для более полной картины принятия решения необходимо подсчитать средний показатель отклонения среднестатистического сотрудника, и, если он окажется сопоставим с показателем 80\%, то выбор в пользу технологий искусственного интеллекта очевиден.

Стоит отметить, что конкурс проходил в середине 2018 года. За два года технологии искусственного интеллекта сделали еще несколько серьезных шагов вперед. Учитывая практически массовый переход банков на ускоренное принятие решения о выдаче кредита, вплоть до нескольких минут, можно сделать вывод, что машинные алгоритмы вытесняют человек $[9,18,19]$.

Данный конкурс является метрикой стадий цифровой трансформации банковского сектора. Аналогичных конкурсов от других банков на соревнованиях, сопоставимого с Kaggle и меньшего уровня, проводилось несколько десятков. Один из последних - на платформе booster.pro от Альфа-банка (сроки проведения: 12 декабря 2020 - 20 января 2021).

Анализ других конкурсов платформы Kaggle и аналогичных ресурсов за последние пять лет показал интерес банков к таким задачам как:

- предсказание транзацкии, которую выполнит клиент в ближайшее время (банки: Banco Santander, Альфа-банк и др);

- выявление методами машинного обучения мошеннических транзакций по кредитным картам;

- предсказание поведение клиента в мобильном приложении (Альфа-банк)

- предсказание стоимости недвижимости (Сбербанк).

Из перечисленных конкурсов можно сделать вывод о направлениях развития цифровой трансформации банковского бизнеса: внедрение технологий искусственного интеллекта для анализа данных по транзакциям для понимания пользовательских предпочтений, повышение безопасности банковских платежей, интеллектуальный цифровой скоринг. Также очевидно изменение стратегии некоторых крупных банков и переход на ниши, не свойственные ранее классическим банкам. 
На платформе Kaggle проводятся конкурсы, имеющие и общецивилизационный эффект. Например, конкурсы по компьютерному зрению: распознавание лиц, распознавание документов и др. Эффект от развития технологий сверточных нейронных сетей $[2,10]$ также затронул бизнес-процессы банковского сектора.

Рассмотренные выше конкурсы были направлены на обработку аналитической информации. Сам процесс принятия решения на подготовленных структурированных данных - это лишь часть любого процесса в банке. Например, для процесса кредитования.

Этапу принятия решения предшествует процесс сбора данных. Здесь неоценимую роль сыграли технологии компьютерного зрения.

Сегодня многие банки предоставляют услугу онлайн оформления кредита (или открытия расчетного счета). Для этого требуется загрузить пакет снимков документов и сделать фотографию своего лица на камеру телефона/ноутбука. Далее алгоритмы компьютерного зрения проанализируют полученные снимки, определят по ним ключевые данные, (номер, серия, дата выдачи и др.), сравнят фотографии на документах и фотографии, полученные в онлайн режиме с камеры, и примут решение, загрузил ли человек действительно свои документы, и являются ли они действующими и легальными.

Конкурсы платформы Kaggle можно рассматривать как хронологию развития технологий искусственного интеллекта в различных отраслях, в том числе и банковского дела.

\section{Заключение}

Технологии искусственного интеллекта являются одними из ключевых в цифровой трансформации банковского сектора.

Банковская отрасль переживает стремительную цифровизацию, в том числе, и за счет технологий искусственного интеллекта.

Хронологию развития технологий искусственного интеллекта можно оценивать по постановке задач и результатам их решений в международных конкурсах по программированию. 
Таким образом, можно сделать вывод о подтверждении гипотезы об использовании задач и результатов конкурсов соревнований по программированию в сфере искусственного интеллекта по задачам банковского сектора, для корреляции этапов цифровой трансформации бизнес-процессов банковской отрасли.

\section{Список литературы}

1. Генеральницкая Е.И. Тенденции трансформации экономических отношений в условиях цифровой экономики (на примере кредитных организаций и предпринимательского сектора) // Наука Красноярья. 2019. Том 8, № 4. С. 20-33.

2. Гудфеллоу Я. Глубокое обучение = Deep Learning / Гудфеллоу Я, Бенджио И., Курвилль А. М.: ДМК Пресс, 2017. С. 652.

3. Долженко Р.А., Бакаленко А.В. Краудсорсинг как инструмент мобилизации интеллектуальных ресурсов: опыт использования в Сбербанке России // Российский журнал менеджмента. 2016. Том 14, № 3. C. 77-102. DOI: 10.21638/11701/spbu18.2016.305

4. Зверькова Т.Н. Региональные банки и Fintech: противостояние или партнерство // Финансы и кредит. 2018. Том 24, № 12 (780). С. 2771 2782. DOI: $10.24891 /$ fc. 24.12 .2771

5. Кириллов В.Н. Искусственный интеллект и глобальные вызовы экономического роста // Шаг в будущее: Искусственный интеллект и цифровая экономика: материалы I Междунар. науч.-практ. конф. Вып. 1. М.: Изд. дом ГУУ. С. 122-127.

6. Котляров И.Д. Типовые бизнес-стратегии участников финансового рынка в условиях финансово-технологической революции // ЭКО. 2019. № 2 (536). С. 135-152.

7. Котляров И.Д. Цифровая трансформация финансовой сферы: содержание и тенденции // Управленец. 2020. Том 11, № 3 (536). С. 72-81. DOI: 10.29141/2218-5003-2020-11-3-6

8. Савина Т.Н. Цифровая экономика как новая парадигма развития: вызовы, возможности и перспективы // Финансы и кредит. 2018. Т. 24, No 3. C. 579-590. https://doi.org/10.24891/fc.24.3.579

9. Самусева С., Буйлов М. Банки уходят в онлайн // Газета Коммерсант. 2020. №48 (6769). С. 8 
10. Сверточная нейронная сеть, часть 2: обучение алгоритмом обратного распространения ошибки [Электронный ресурс] // MS: [сайт]. Режим доступа: https://habr.com/ru/post/348028

11. Смирнов Е.Н., Лукьянов С.А. Формирование и развитие глобального рынка систем искусственного интеллекта // Экономика региона. 2019. Т. 15. Вып. 1. С. 57-69. DOI: 10.17059/2019-1-5

12. Attention Is All You Need. Ashish Vaswani, Noam Shazeer, Niki Parmar, Jakob Uszkoreit, Llion Jones, Aidan N. Gomez, Lukasz Kaiser, Illia Polosukhin. [Электронный ресурс] // MS: [сайт]. Режим доступа: https://arxiv.org/abs/1706.03762

13. Breidbach C., Keating B., Lim C. Fintech: research directions to explore the digital transformation of financial service systems // Journal of Service Theory and Practice. 2019. Vol. 30 No. 1, pp. 79-102. https://doi. org/10.1108/JSTP-08-2018-0185

14. Knewtson H., Rosenbaum Z. Toward understanding FinTech and its industry // Managerial Finance. 2020. Vol. 46 No. 8, pp. 1043-1060. https:// doi.org/10.1108/MF-01-2020-0024

15. LeCun Y., B. Backpropagation Applied to Handwritten Zip Code Recognition / Y. LeCun, B. Boser, J. S. Denker, D. Henderson, R. E. Howard, W. Hubbard and L. D. Jackel // Neural Computation, 1(4):541-551, Winter 1989.

16. Romanova I., Kudinska, M. Banking and Fintech: A Challenge or Opportunity? // Contemporary Studies in Economic and Financial Analysis. 2016. Vol. 98, pp. 21-35. https://doi.org/10.1108/S1569-375920160000098002

17. Sepp Hochreiter, Jürgen Schmidhuber. Long short-term memory // Neural Computation November 15, 1997, Vol. 9, No. 8, pp. 1735-1780. DOI: 10.1162/neco.1997.9.8.1735. https://web.archive.org/web/20150526132154/ http://deeplearning.cs.cmu.edu/pdfs/Hochreiter97_lstm.pdf

18. Shiau W., Yuan Y., Pu X., Ray S. Understanding fintech continuance: perspectives from self-efficacy and ECT-IS theories // Industrial Management \& Data Systems. 2020. Vol. 120 No. 9, pp. 1659-1689. https:// doi.org/10.1108/IMDS-02-2020-0069

19. Singh S. What drives FinTech adoption? A multi-method evaluation using an adapted technology acceptance model // Management Decision. 2020. Vol. 58 No. 8, pp. 1675-1697. https://doi.org/10.1108/MD-09-2019-1318 


\section{References}

1. General'nickaja E.I. Tendencii transformacii jekonomicheskih otnoshenij v uslovijah cifrovoj jekonomiki (na primere kreditnyh organizacij i predprinimatel'skogo sektora) [Trends in the transformation of economic relations in the digital economy (on the example of credit institutions and the business sector)]. Nauka Krasnojar'ja. 2019. V. 8, № 4. P. 20-33.

2. Gudfellou Ja.Glubokoe obuchenie [Deep Learning]. M.: DMK Press, 2017. P. 652

3. Dolzhenko R.A., Bakalenko A.V. Rossiyskiy zhurnal menedzhmenta. 2016. V. 14, № 3. P. 77-102. DOI: 10.21638/11701/spbu18.2016.305

4. Zver'kova T.N. Finansy i kredit. 2018. V. 24, № 12 (780). P. 2771-2782. DOI: $10.24891 /$ fc. 24.12 .2771

5. Kirillov V.N. Iskusstvennyj intellekt i global'nye vyzovy jekonomicheskogo rosta [Artificial intelligence and global challenges of economic growth]. Shag v budushhee: Iskusstvennyj intellekt i cifrovaja jekonomika: materialy I Mezhdunar. nauch.-prakt. konf. [Step into the Future: Artificial Intelligence and the Digital Economy: Proceedings of the 1st Intern. scientific-practical conf.]. 2017. Issiu 1. M.: Izd. dom GUU. P. 122-127.

6. Kotlyarov I.D. EKO. 2019. № 2 (536). P. 135-152.

7. Kotlyarov I.D. Upravlenets. 2020. Vol, 11, № 3 (536). P. 72-81. DOI: 10.29141/2218-5003-2020-11-3-6

8. Savina T.N. Cifrovaya ekonomika kak novaya paradigma razvitiya: vyzovy, vozmozhnosti i perspektivy [Digital economy as a new para-digm of development: challenges, opportunities, and prospects]. Finansy i kredit. 2018. V. 24, No 3. P. 579-590.

9. Samuseva S, Bujlov M. Banki uhodjat v onlajn [Banks go online]. Gazeta Kommersant. 2020. №48 (6769). P. 8

10. Svertochnaja nejronnaja set', chast'2: obuchenie algoritmom obratnogo rasprostranenija oshibki [Convolutional neural network, part 2: learning by error back propagation algorithm]. https://habr.com/ru/post/348028

11. Smirnov E.N., Luk'janov S.A. Formirovanie i razvitie global'nogo rynka sistem iskusstvennogo intellekta [Form and development of the global market for artificial intelligence systems]. Jekonomika regiona. 2019. V. 15. №. 1. P. 57-69. DOI: 10.17059/2019-1-5 
12. Attention Is All You Need. Ashish Vaswani, Noam Shazeer, Niki Parmar, Jakob Uszkoreit, Llion Jones, Aidan N. Gomez, Lukasz Kaiser, Illia Polosukhin. https://arxiv.org/abs/1706.03762

13. Breidbach C., Keating B., Lim C. Fintech: research directions to explore the digital transformation of financial service systems. Journal of Service Theory and Practice. 2019. Vol. 30 No. 1, pp. 79-102. https://doi. org/10.1108/JSTP-08-2018-0185

14. Knewtson H., Rosenbaum Z. Toward understanding FinTech and its industry. Managerial Finance. 2020. Vol. 46 No. 8, pp. 1043-1060. https:// doi.org/10.1108/MF-01-2020-0024

15. LeCun Y., B. Backpropagation Applied to Handwritten Zip Code Recognition / Y. LeCun, B. Boser, J. S. Denker, D. Henderson, R. E. Howard, W. Hubbard and L. D. Jackel. Neural Computation, 1(4):541-551, Winter 1989.

16. Romanova I., Kudinska, M. Banking and Fintech: A Challenge or Opportunity? Contemporary Studies in Economic and Financial Analysis. 2016. Vol. 98, pp. 21-35. https://doi.org/10.1108/S1569-375920160000098002

17. Sepp Hochreiter, Jürgen Schmidhuber. Long short-term memory. Neural Computation November 15, 1997, Vol. 9, No. 8, pp. 1735-1780. DOI: 10.1162/neco.1997.9.8.1735. https://web.archive.org/web/20150526132154/ http://deeplearning.cs.cmu.edu/pdfs/Hochreiter97_lstm.pdf

18. Shiau W., Yuan Y., Pu X., Ray S. Understanding fintech continuance: perspectives from self-efficacy and ECT-IS theories. Industrial Management \& Data Systems. 2020. Vol. 120 No. 9, pp. 1659-1689. https://doi. org/10.1108/IMDS-02-2020-0069

19. Singh S. What drives FinTech adoption? A multi-method evaluation using an adapted technology acceptance model. Management Decision. 2020. Vol. 58 No. 8, pp. 1675-1697. https://doi.org/10.1108/MD-09-2019-1318

\section{ДАННЫЕ ОБ АВТОРЕ}

Терещенко Сергей Николаевич, заведующий кафедрой «Прикладная информатика», доцент, кандидат технический наук Новосибирский государственный университет экономики и управления 
ул. Каменская, 56, г. Новосибирск, Новосибирская область, 630099, Российская Федерачия

e-mail:sg12@ngs.ru

\section{DATA ABOUT THE AUTHOR}

Tereshchenko Sergei Nikolaevich, Head of the Department «Applied Informatics», Associate Professor, Candidate of Tech. Sciences Novosibirsk State University of Economics and Management 56, Kamenskaya Str., Novosibirsk, 630099, Russian Federation sg12@ngs.ru

ORCID: 0000-0003-2309-8445 